\title{
Information System for Epidemic Control: a computational solution addressing successful experiences and main challenges *
}

Guilherme Conceição Rocha ${ }^{1}$, Henrique Mohallem Paiva ${ }^{2}$, Davi Gonçalves Sanches ${ }^{2}$, Daniel Fiks ${ }^{3}$, Rafael Martins Castro ${ }^{4}$, and Lucas Fernando Alvarenga e Silva ${ }^{2}$

${ }^{1}$ Department of Mechanical Engineering, Aeronautical Institute of Technology (ITA)

Praça Marechal Eduardo Gomes, 50, São José dos Campos, SP, Brazil

${ }^{2}$ Institute of Science and Technology (ICT), Federal University of Sao Paulo(UNIFESP)

Rua Talim, 330, São José dos Campos, SP, Brazil

${ }^{3}$ Santa Marcelina School of Medicine (FASM)

Rua Cachoeira Utupanema, 40 - São Paulo - SP, Brazil

${ }^{4}$ Leonardo da Vinci University (Uniasselvi)

Avenida Fernando Corrêa da Costa, 374, Cuiabá, MT, Brazil

\footnotetext{
${ }^{*}$ This is a preprint of the following paper:

Rocha, G.C., Paiva, H.M., Sanches, D.G., Fiks, D., Castro, R.M. and Silva, L.F.A. (2021), "Information system for epidemic control: a computational solution addressing successful experiences and main challenges", Library Hi Tech, Vol. ahead-of-print No. ahead-of-print. https://doi.org/10.1108/LHT-11-2020-0276
}

This is an author-created, un-copyedited version of an article accepted for publication in Library Hi Tech. 


\section{Abstract}

\section{Purpose}

The SARS-CoV-2 pandemic has caused a major impact on worldwide public health and economics. The lessons learned from the successful attempts to contain the pandemic escalation revealed that the wise usage of contact tracing and information systems can widely help the containment work of any contagious disease. In this context, this paper investigates other researches on this domain, as well as the main issues related to the practical implementation of such systems, and specifies a technical solution.

\section{Methodology}

The solution is based on the automatic identification of relevant contacts between infected or suspected cases with susceptible people; inference of contamination risk based on symptoms history, user navigation records, and contact information; real-time georeferenced information of population density of infected or suspect people; and automatic individual social distancing recommendation calculated through the individual contamination risk and the worsening of clinical condition risk.

\section{Findings}

The solution was specified, prototyped, and evaluated by potential users and health authorities. The proposed solution has the potential of becoming a reference on how to coordinate the efforts of health authorities and the population on epidemic control.

\section{Originality}

This paper proposed an original information system for epidemic control, which was applied for the SARS-CoV-2 pandemic and could be easily extended to other epidemics.

\section{Keywords}

COVID-19, SARS-CoV-2, contact tracing, information systems, contamination risk, social distancing 


\section{Introduction}

The COVID-19 pandemic, which is the disease caused by the Acute Respiratory Syndrome Coronavirus 2 (SARS-CoV-2), has impacted every country around the globe, leading to social restrictions, economic recession, and more than 1.5 million deaths to this point. The transmissibility rate of this new virus is higher than its predecessors and much research has focused on how human mobility can help to spread infections (Abebe et al., 2020). Many governments have imposed social distancing policies aiming to control the increase in cases and to reduce death tolls, although those measures eventually led to a great economic recession. In emerging market economies, such as Brazil, this economic impact is critical, since the socioeconomic disparities can be intensified and more people will likely suffer from unemployment, poverty, and hunger (Aquino et al., 2020; Storopoly et al., 2020).

To mitigate the economic impacts of social distancing, mathematical modeling and solutions in informatics have been used to provide governors a better understanding of the pandemic's dynamic, which could orientate less restrictive and more efficient social distancing measures (Nicola et al., 2020; Kucharski et al., 2020; Navarro-Alamán et al., 2020). In Brazil, a country with continental dimensions where 217 million people live under great socioeconomic inequalities, updated data on new cases, new deaths and human mobility is a major challenge for municipal, state, and federal governors (Aquino et al., 2020). Each Brazilian region has been affected by the pandemic differently, and the disease's dynamics varied in time and space during the outbreak (Aquino et al., 2020). Those differences can be better described by updated data on mobility (Kucharski et al., 2020).

Smart device applications can be used for contact tracing and user location monitoring to provide more accurate data on human mobility (Rathbone et al., 2017). They can also be used as a health information platform that is able to inform the user's infection or complication risks based on personal data, to guide about the best safety behaviors, as well as to inform health professionals and public agents about the current epidemiological situation in the community and health institutions (Rathbone et al., 2017). In Brazil, it is estimated that around $80 \%$ of the population access the 
internet from smartphone devices, showing how reasonable this approach is (IBGE, 2018). Thus, this project describes an app that provides updated data on mobility to allow contact tracing of new cases and the generation of heat maps with health color codes, which are important and necessary tools for the Brazilian governors to better orientate social distancing measures and to mitigate the COVID-19 impacts in different regions. Most importantly, all the app features here described can be easily adapted for other epidemics, such as Dengue, Zika Virus, among others, that affect Brazil and many other countries and impose life and economic losses; therefore, the application proposed here is not restricted to the COVID-19 pandemic and can be a useful public health tool to combat other diseases.

\section{Literature Review}

The first case of COVID-19 infection, the disease caused by the Acute Respiratory Syndrome Coronavirus 2 (SARS-CoV-2), was notified to the World's Health Organization (WHO) on December 31st, 2019. On March 11th, 2020, a few months later, WHO decreed COVID-19 as a global pandemic, alerting about its high transmissibility and the socioeconomic impacts of the disease (Abebe et al., 2020).

Initially understood as a respiratory disease, review studies have concluded that COVID-19, in its most severe cases, presents itself as a systemic infection. COVID-19 causes different symptoms such as cough, fever, shortness of breath, diarrhea, nausea, and other gastrointestinal symptoms and neurological problems, among others (D'Amico et al., 2020; Hu et al., 2020; Khai and Kai, 2020; Parasa et al., 2020). Furthermore, COVID-19 infection leads to pneumonia in severe cases, and patients often need the help of ventilators to breathe properly (Iyengar et al., 2020).

In Brazil, the largest country and the greatest economy in Latin America, with a total population of 217 million people, the social disparities and vulnerabilities present challenges to governments regarding testing, contact tracing, social distancing measures, and treatment of all COVID-19 patients. By June 2020, Brazil was already among the four most impacted countries by the pandemic 
globally while having one of the highest underreport tolls of cases and deaths (Storopoly et al., 2020).

Continental dimensions and the different socioeconomic characteristics of Brazilian regions led to different disease dynamics across its territory. These characteristics would require a strong integrated national policy to tackle the pandemic effectively, but much research has shown that the unexistence of a coordination plan between the federal and the estadual governments represented a problem to face the rapid increase in cases and deaths in the country, including the denial of the virus' threat by the federal authorities (Aquino et al., 2020; Stotopoli et al., 2020). Recognizing the high health threat that COVID-19 represents is important for the adoption of safety behaviors by the population since studies have shown that trust has a significantly positive effect on health knowledge adoption when there is a high health threat (Huo et al., 2017).

Collecting real-time data can be very helpful while approaching some problems, including the ones in the healthcare field (Kumar and Mostafa, 2020). One way to collect those data is by using applications in smart devices, like smartphones. Informatics has been proposed and used as a solution to many different health care challenges, like monitoring cancer survivors through a gamified mobile app (Navarro-Alamán et al., 2020); prescreening systems with smart wearables to detect abnormal respiratory events (Camc1, Ersoy and Kaynak, 2019); computer-assisted decision support systems in pulmonary cancer detection and stage classification on CT images (Masood et al., 2018); sustainable collaborative consumer health applications for self-management of chronic diseases (NavarroAlamán et al., 2020); and efficient handling of the patient information in an intensive-care unit (Newman et al., 2020). A systematic review of big data handling in the healthcare applications provided a comprehensive and detailed study of machine learning, cloud-based, heuristic-based, agent-based, and hybrid mechanisms for solving problems (Pashazadeh and Navimipour, 2018). This review showed that several papers presented positive effects on behavior change, decision, and public health policymaking, which indicates how successful the informatics approach can be while solving those challenges. The challenges involved in the design and use of large-scale health information systems have been thoroughly reviewed by the survey presented in (Sligo et al., 2017). 
Informatics not only helps collect data to create public policies but also to inform people based on those data. Research on how consumers use online health information as a learning process elucidated how people choose different platforms to search for information and how they progress during this task. This study by Chen et al. (2020) showed that the type of subject and the user's socioeconomic and academic backgrounds influence the quality of the search, indicating that some people might take longer to find relevant and true information about different health topics. In this case, smartphone applications can be a helpful tool to inform consumers about their health risks and to give orientation on self-care behaviors in a fast, safe, and easy-to-learn environment (Rathbone et al., 2017), especially in countries with lower socioeconomic indicators: A study comparing how university students from South Africa and Finland sought web-based health information showed that the South African students found information online more passively and often opted for social networking sites instead of search engines, which generally provides less accurate information, whereas Finnish students chose search engines more frequently. Brazil shares similar socioeconomic disparities with South Africa, indicating that providing more accurate health information in friendlier and widely used platforms can have a good impact on the communication of health information (Rantala et al., 2019).

Some countries, like China and South Korea, have been very successful at sustaining epidemic suppression using mobile phone apps. Since transmission of coronaviruses can happen from symptomatic, pre-symptomatic, and asymptomatic infected individuals, there are two possible interventions: (i) isolation of symptomatic cases and (ii) tracing the contacts of symptomatic cases and quarantining them. The delay between the confirmation of a new case and the trace of its contacts must be avoided so that the epidemic can be controlled (Ferretti et al., 2020).

In China, the use of an app as a public health policy helped the government to not only quarantine symptomatic individuals, but also to trace their recent contacts and quarantine them. By doing so, China could reduce the thousands of infections per day in early January to less than 150 infections per day in early March, demonstrating contact tracing effectiveness (Ferretti et al., 2020). Another 
possible tool to monitor the evolution of an epidemic is the heat map. Spatial-temporal heat maps were successfully used in Singapore (Jiang et al., 2020).

Ferretti et al. (2020) concluded that digital contact tracing would assist in SARS-CoV-2 epidemic control. To quantify this phenomenon, they developed a mathematical model for COVID-19 infectiousness, considering the prevalence of pre-symptomatic, symptomatic and asymptomatic individuals, as well as the estimate of environmentally mediated transmission via contamination.

The model expresses that the infectiousness varies as a function of time since infection. Therefore, early detection of possible infected individuals and subsequent isolation of them is crucial to reduce infectiousness, promoting epidemic control.

All technological applications that attempted to contain the COVID-19 pandemic from spreading had at least one of the following features, as it can be noticed on the survey presented in (Mao, Jiang and Nametz, 2020): automatic detection of close contacts between individuals, using Bluetooth Low Energy (BLE) technology (BLE Contact Tracing), as adopted by Singapore, in the solution named TraceTogether (Singapore Ministry of Health, 2020); usage of GPS signal to estimate user proximity (GPS Contact Tracing), as adopted by Norway, in the solution named Smittestopp (Nikel, 2020); continuous monitoring of users' location by wristbands to strictly enforce 14-days quarantine for airport arrivals (User Location Monitoring), as adopted by Hong Kong, in the solution named StayHomeSafe (Hong Kong Government, 2020); usage of GPS signal to alert users when they come within 100 meters of a location visited by an infected person, and to exhibit a heat map of infection density close to the user's location (Infection Heat Map), as adopted by South Korea, in the solution named Corona 100 (Dudden and Marks, 2020); usage of health survey based on symptoms and patient risk factors to define a health colored code (Health Color Code), as adopted by China in the Chinese health code system (Chinese Government, 2020). In summary, the foundations of all solutions are based on the combination of the following features: Contact Tracing; User Location Monitoring; Heat Map; and Health Color Code.

On the other hand, the available solutions are based on assumptions that do not hold true for low and middle-income countries, such as Brazil, which face challenges such as low budget and logistical 
difficulties; limited access to smartphones from a significant part of the population; limited healthcare resources; low availability of tests, and so on.

Ferretti et al. (2020) demonstrated that efficiency in fighting against COVID-19 depends on how fast the reaction from initiating symptoms to case isolation and quarantine of contacts would be. Considering an automatic contact tracing solution and one day delay from symptoms to isolation and quarantine, and a success rate of quarantining contacts of $70 \%$, the authors estimated epidemic control with a success rate of $30 \%$ in isolating cases. The implication of that is that automatic contact tracing solution needs to be well spread among the population to be effective.

Also, Levy (2020) highlighted some concerns on adopting decentralized digital contact tracing model solutions in the United Kingdom. Main concerns are: (i) decentralized solutions depend on authentication of clinical tests, jeopardizing isolating cases speed; (ii) decentralized systems seem to introduce delays in the reporting of symptoms; (iii) a decentralized model allows only 'risk to' an individual from their exposures to be understood, without providing enough stimulus for an individual to self-diagnose. In Brazil, these concerns are even greater due to the social disparities and the larger territory and population.

In this research, CoronaTrack, an application characterized as an information system about the COVID-19 pandemic, has been developed. CoronaTrack adopts a semi-centralized architecture where users would report symptoms voluntarily, check their infection risk estimate based on habits and navigation risk (without depending only on the contact tracing feature), navigate on a heat map of infected people density without depending on a high adherence of the population to the app, and receive calls from a health agent to perform test, receive teleorientation or vaccine. This approach represents a low-cost solution that enables healthcare systems to rapidly update relevant data on cases, testing, and contact tracing, as well as making individuals more aware of their risks and responsibilities as regards the COVID-19 pandemic, creating more resilient communities. Even though the final product is specific to COVID-19, it can be easily extended to other epidemic diseases. 


\section{Materials and Methods}

This section presents some insights and steps on the usual application development process that allow fast and assertive critical decision making while keeping the consistency and robustness of the desired final application. The application described here can be generalized to fight against other pandemic or epidemic diseases.

\section{General Methodology}

As one can notice, according to the literature review, the foundations of existing app solutions to fight against COVID-19 are based on the combination of the following features: Contact Tracing; User Location Monitoring; Heat Map; and Health Color Code. Most of these solutions adopted a decentralized digital contact tracing model solution, enhanced with other features.

On the other hand, Levy (2020) highlighted concerns on adopting such decentralized solutions due to dependence on clinical test results and not providing enough stimulus for an individual to selfdiagnose. Therefore, for countries like Brazil, it is recommended that digital contact tracing solution architecture be semi-centralized and based on self-diagnose features.

In this context, the research question that guided our research was: Is it possible to conceive and validate a semi-centralized digital contact tracing low-cost solution that would consider the user's willingness, user's recent automatically detected contacts and navigation records to infer user infection risk and use this information together with other features to maximize healthcare system ability in combating the COVID-19 outbreak?

The applied methodology to answer this question is detailed in the steps below:

Step 1: Define a semi-centralized architecture able to process both user personal data (habits, symptoms reported voluntarily, and navigation records) with other users' anonymized data (contacts records, clinical test results, and infection risk) 
Step 2: Define a process to estimate user infection risk based on the combination of user personal data, the recent user automatically detected contacts and navigation records.

Step 3: Recommend a social distancing rigor based on the combination of user infection risk and individual mortality risk.

Step 4: Specify a set of features to promote better communication between users and health agents, and to improve visibility of COVID-19 outbreak for the health system to optimize public effort in providing teleorientation, monitoring patients, performing clinical tests, and prioritizing users that need to be vaccinated first.

Step 5: Prototype the solution using an agile product development process.

Step 6: Validate the solution among stakeholders.

These steps will be detailed in the following sections.

\section{Goals and Requisites of the Application}

The application is expected to get user information, like comorbidities, addictions, medications, hygienic habits, COVID-19 test results, user contacts and user localization. The application shall analyze the data and notify each user calculated risk in colors (Health color code). The health color code is associated with the social isolation rigor recommended for the user and can assume three different status: "green", "yellow", and "red" as detailed in section 2.2.

The contact tracing feature uses the Bluetooth signal to discover other smartphones Bluetooth, exchange Random Temporary Exposure keys, and Rotating Proximity identifiers with them. These contact logs, firstly stored on each device, will be shared on the cloud to discover possibly exposed users who have had contact with infected individuals. CoronaTrack application also shows the density of infected or suspected to be infected people in a georeferenced region by Heat Maps. This feature uses information obtained from the users and public health databases. All the app expected features and the relationships among them are described in Figure 1. The development team 
guarantees the security of the databases employing well-known cloud services and data protection techniques.

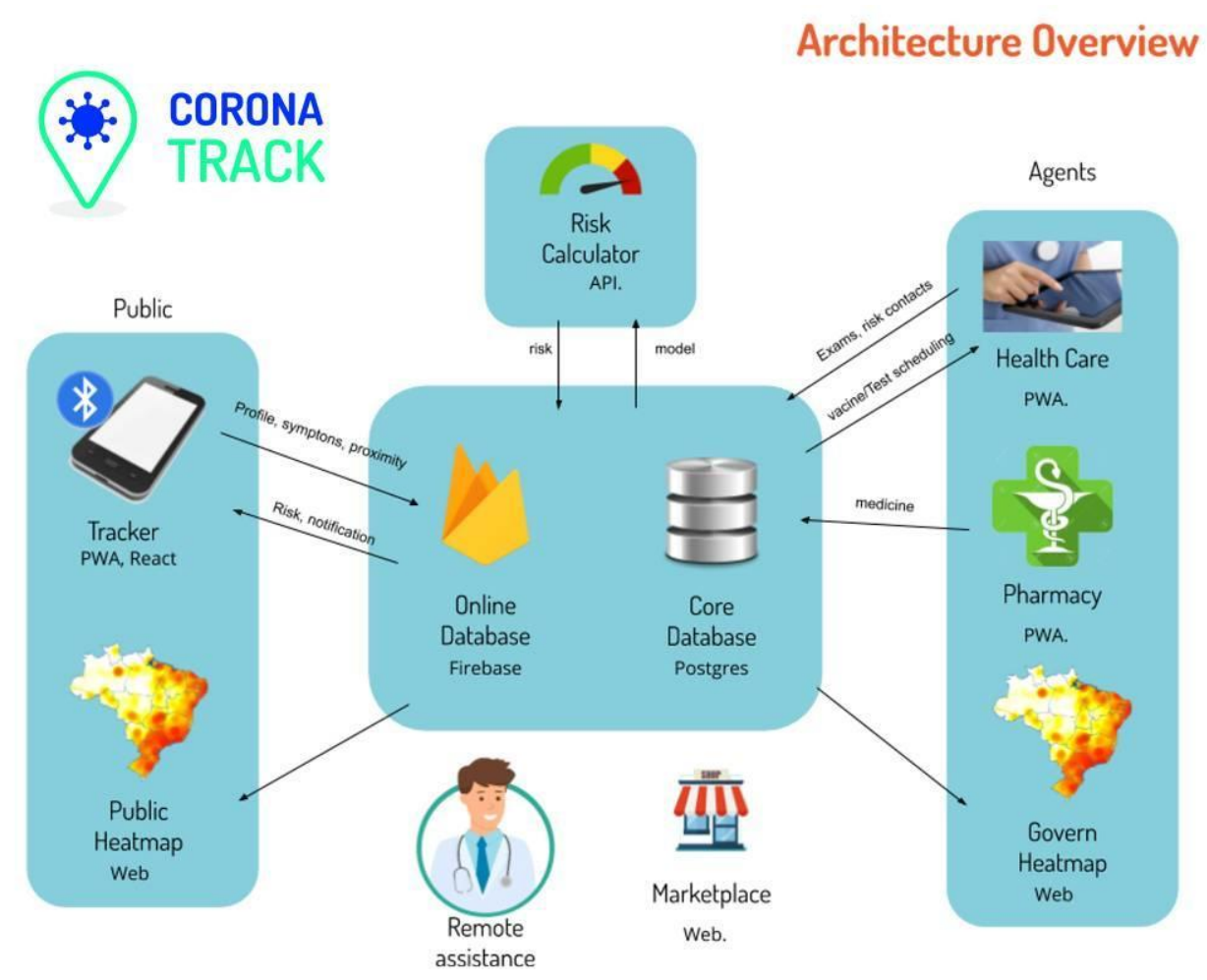

Figure 1 - Overview of the CoronaTrack app architecture.

\section{Health Color Code estimate}

The health color code corresponds to the level of social distancing suggested for the user. In the CoronaTrack solution, three different colors were considered, with different levels of social distancing recommendation. These colors were chosen in accordance with the "Risk Classification" used by the public health system in Brazil.

- "Green" - individuals identified as healthy and out of the high mortality risk group.

They can perform productive activities and access non-essential services. 
- "Yellow" - individuals with light or moderate suspicion of being infected and out of the high mortality risk group. They should not perform productive activities and should only access essential services.

- "Red" - individuals which are infected, have high suspicion of being infected, or pertain to the high mortality risk group. They should be completely isolated from any kind of social interaction.

One can estimate the health color code by the combination of the individual mortality risk based on pre-existing health conditions or chronic diseases, and the risk of the individual being infected (infection risk).

The list of pre-existing health conditions or chronic diseases that increase the mortality risk due to SARS-CoV-2 infection was based on a Brazilian Health Ministry document (2020). On the other hand, the infection risk is determined based on the following parameters:

a) Recent contact history between the user and individuals that are infected or suspect to be infected, automatically identified through contact tracing feature;

b) User navigation records at hot and warm zones on the heat map;

c) Preventive profile score, determined based on mobility and personal hygiene habits;

d) Symptoms in recent records.

Table 1 summarizes how the health color code is estimated. 
Table 1 - Health color code as function of infection and mortality risks

\begin{tabular}{|c|c|c|}
\hline Infection risk & Mortality risk & Health color code \\
\hline Low & High & RED \\
\hline Moderate & High & RED \\
\hline High & High & RED \\
\hline Low & Low & GREEN \\
\hline Moderate & Low & YELLOW \\
\hline High & Low & RED \\
\hline
\end{tabular}

User's privacy

User's privacy is a main concern in the development of contact-tracing apps (Bradford et al., 2020; Zastrow, 2020). To comply with Brazilian user privacy protection law, the solution presented here performs Contact Tracing using Bluetooth Low Energy (BLE) technology and does not implement any kind of user location monitoring that may disclose user georeferenced position. For this same reason, the heat map feature had its precision degraded to 400 meters, to avoid disclosure of individuals and commercial establishment locations. 


\section{Architecture and implementation}

The architecture and implementation of the CoronaTrack Application is described in detail in the Appendix 1, published online.

\section{Development and Tests}

CoronaTrack was developed by using Scrum, an agile approach for project management that embraces higher rates of change (Williams and Cockburn, 2003). Despite CoronaTrack critical features, an agile methodology was selected because CoronaTrack had a short period to be developed.

Since the project management is agile, to ensure the accelerated implementation of the product, the development team should ensure the correctness of each step of the application by their critical scope. To accomplish this recommendation, an experienced test team was selected to ensure development correctness.

The visual interface and user journey for the CoronaTrack application were designed on Figma (Figma, 2020). The CoronaTrack app was divided into two parts: default user application and government health authority's application. Default user application was implemented with the React Native Framework on JavaScript programming language. Continuous integration/continuous delivery $(\mathrm{CI} / \mathrm{CD})$ tools with unit tests were used for the integration and functional tests were performed at the final regular user application implementation.

The user interface (UI) and user experience (UX) of both applications were validated by a multidisciplinary team composed of a sample of regular users and government health authorities.

Government health authorities' application has not been implemented yet. It is still a design prototype at FIGMA. 


\section{Results}

The CoronaTrack solution was developed to reach most of the population. The application is available on Android and iOS smartphones. The final users are either regular users or health agents. From regular users, the application collects georeferenced, contact tracing, and personal data to analyze and return their COVID-19 infection risk, quarantine information and georeferenced density of infections. The app helps health agents to make better decisions, delivering epidemiological profiles of the pandemic and information about the infections to the health systems.

The app pages were validated by an UX/UI team and by the Health's Secretary of the Brazilian city of São José dos Campos - SP.

In the following, details of the app navigation for regular users and health agents are presented.

\section{Regular User: Starting the navigation}

Initially, the user sees informational pages describing application goals and features, as well as all information that will be acquired, in compliance with the general data protection law. Afterwards, the user is directed to the login page. If this is their first access, the user is asked to fill two mandatory forms to continue using the app: (i) The first form contains personal information, such as documents, name, gender, birth date, etc; (ii) The second form asks if the user has taken any COVID-19 tests: if positive, the app asks when the respective test was taken and its result; if false, the app asks about possible contacts with infected people. After that, the user is directed to another form, addressing hygienic habits, comorbidities, medicines, vaccines, etc.

Some of the pages about the COVID-19 test journey and the login page are illustrated in Figure 2. In Figure 2(a), the user writes his/her email and password to login or to sign in, if this is his/her first access to the app; in (b), the user is asked if they have ever been tested for COVID-19. This question directs to two different approaches since the user can be a confirmed case or a suspected case, depending on the answer; (c) and (d) show pages in case the user's answer for the last question is 
"no": in (c), according to the WHO isolation period recommendations for infected people, the app asks if the user knows about possible contacts with infected individuals over the last 14 days, since those cases could potentially spread the virus to others; in (d), the user is asked about contact with suspect cases of COVID-19 over the last 14 days, since those people could infect others during this period; the pages represented in (e) and (f) aim to investigate how much social distancing the user has been practicing: in (e), the app asks how often the user leaves the house a week and for what reasons they do so. This is important because essential service workers and people in more economically vulnerable conditions cannot stop going out to work; in (f), the app asks how the user practices social distancing when they go out because, while some people are able to do it, others might not due to work or public transport conditions. The WHO recommends that people keep protective measures for COVID-19 as much as possible when they leave the house. 
(a)

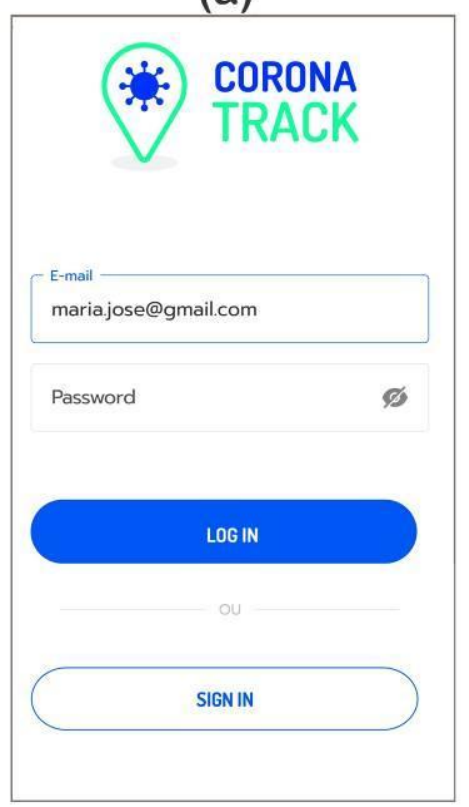

(d)

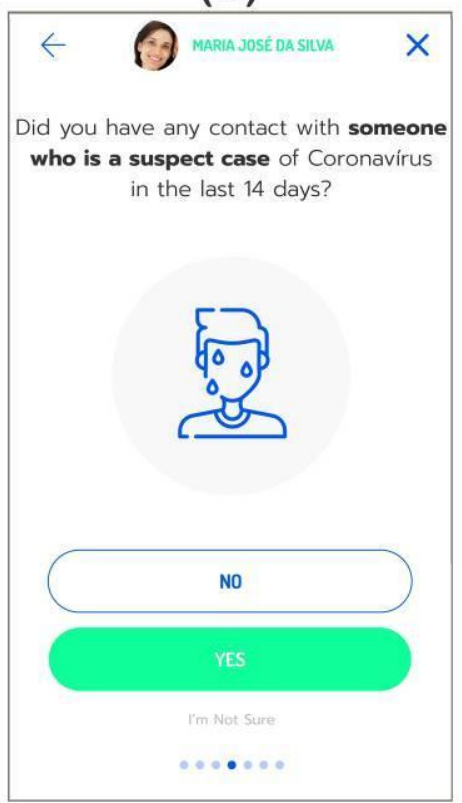

(b)

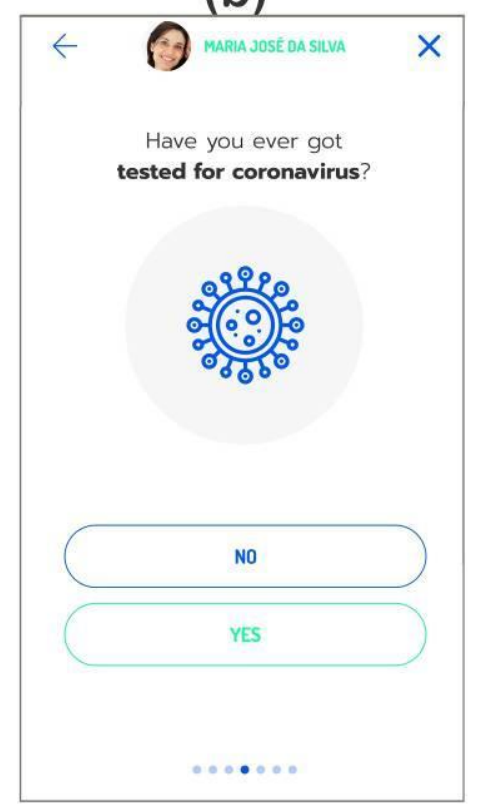

(e)

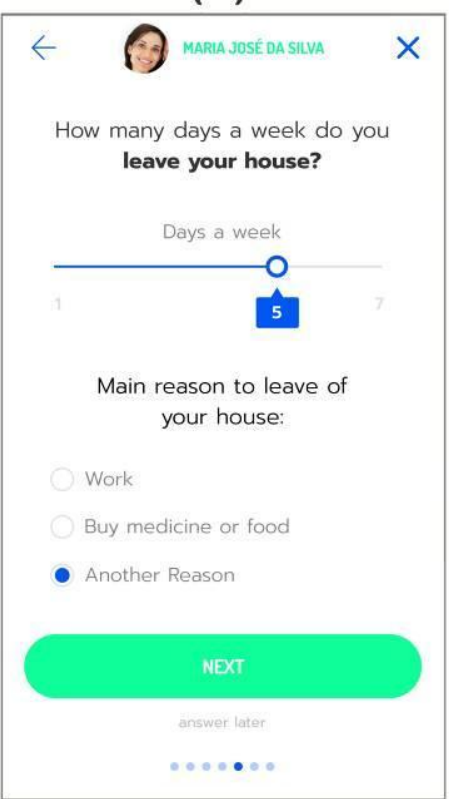

(c)

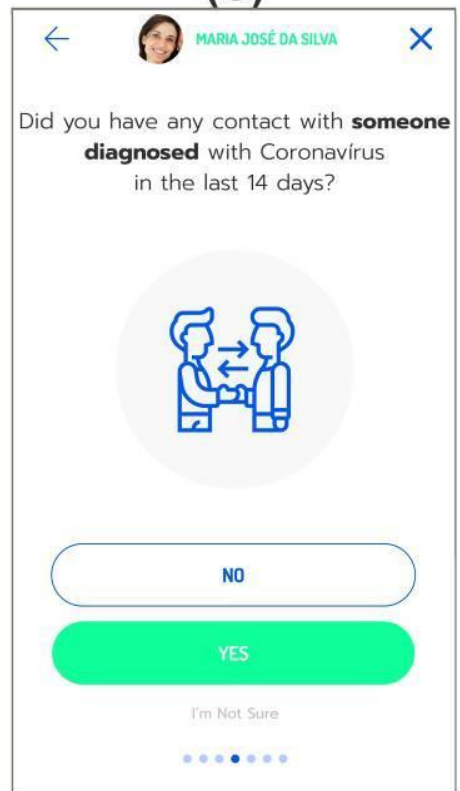

(f)

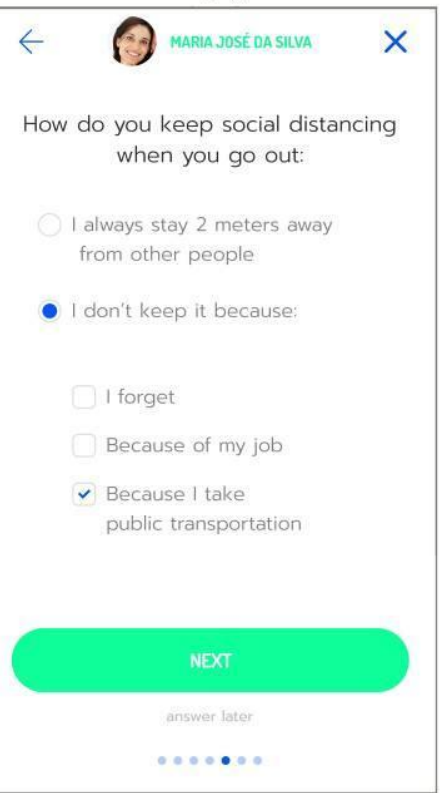

Figure 2 - First pages of the application ${ }^{\dagger}$. (a) App’s login page; (b) Form with the second page, asking if the user has taken any COVID-19 tests; (c,d) pages shown in case of "no" on the second page, asking if the user have had any contact with confirmed or suspected infectious individuals; (e), (f) pages asking about user habits.

${ }^{\dagger}$ The user's picture in Figures 2 to 5 is from a public database, licensed under a Creative Commons Public Domain Mark license. 
The default user is then conducted to the main page, illustrated in Figure 3(a), where he/she can access the following actions: Profile Information; My Health; Tele Orientation; Public Utility; Settings; Log Out.

- The Profile information page, in Figure 3(b,c,d), shows the user's risk. This information intends to inform the user about what measures should be taken depending on how great their risk of infection is. This feature aims to direct people to make better choices to prevent new cases and help public policies of contact tracing

- $\quad$ My Health page (Figure 3(e)) lets the user report symptoms or their COVID-19 test result. If a user reports a true result, all his contacts traced by the contact trace feature have their profile risk updated. This is important for updated data about suspect cases will help public agents to trace contacts and take better decisions to isolate cases.

- Tele Orientation is not CoronaTrack purpose, so this option redirects the user to a third-party application.

- Public Utility contains the heat map as shown in Figure 3(f), which indicates near locations based on georeferenced data and their respective density of infections to default users. This tool is very helpful to inform users about regions with greater risk of infection because it is a graphic tool that shows streets, neighbors and public places in the city. These heat maps have low accuracy to preserve sensitive users' information. 
(a)

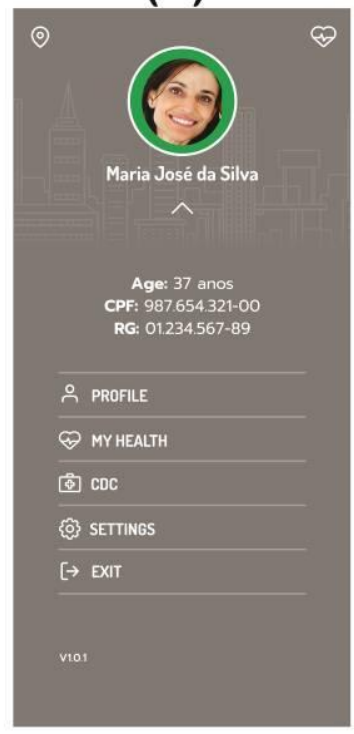

(d)

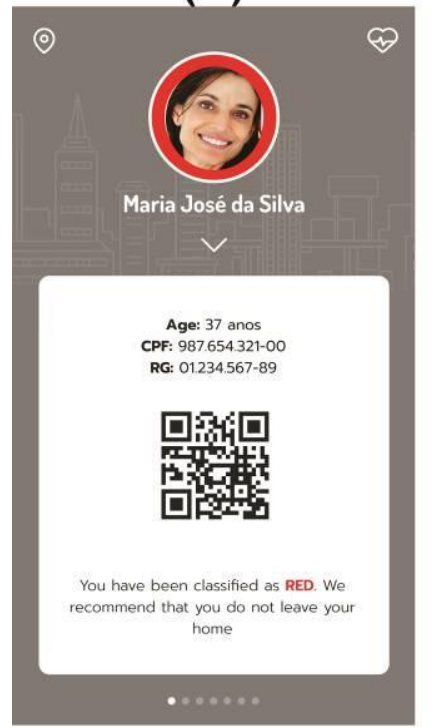

(b)

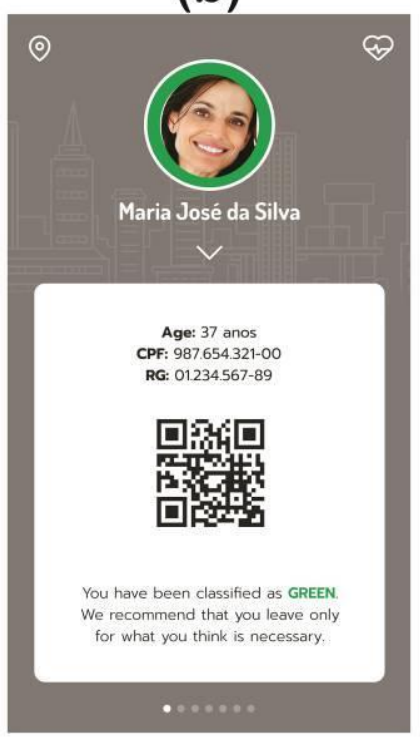

(e)

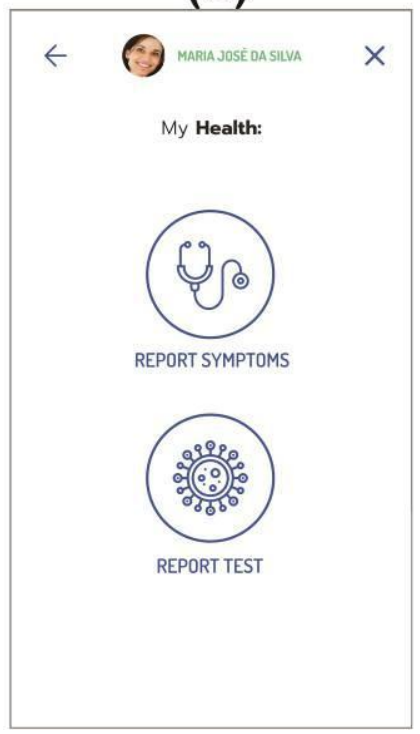

(c)

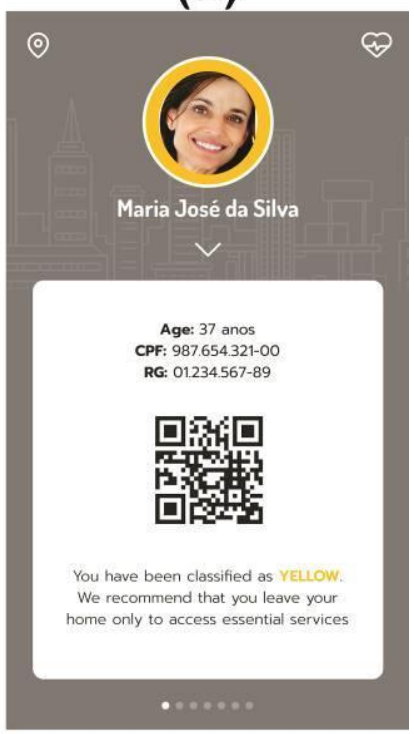

(f)

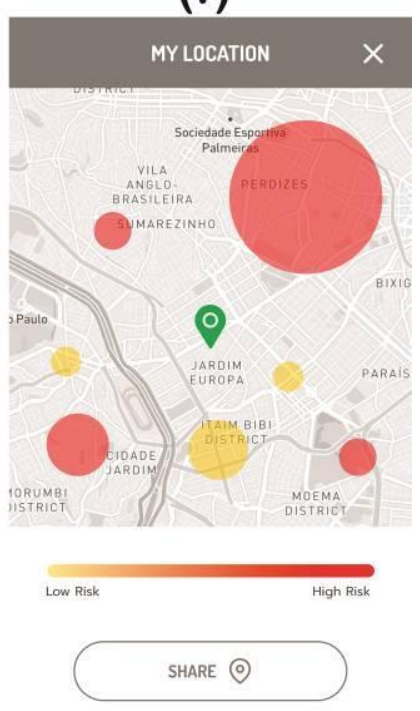

Figure 3 - (a) Main page of the CoronaTrack application, with all the default users actions; (b,c,d) "Profile Information" action, with the color changing accordingly to the profile risk; (e) "My Health" action, allowing the user to report symptoms or a COVID-19 test result; (f) Heat Map feature with COVID-19 density of infections. 
Regular User: The "Profile Information" Action

Profile pages also present some information about the user profile risk. This information is divided into infection risk and mortality (complication) risk, and presents what the user can/cannot do, according to the Ministry of Health. Some of these pages are demonstrated in Figure 4.

In Figure 4 (a), a green profile is shown representing a low risk of infection. This result tells the user that he or she has not been in contact with suspected or infected individuals, which means that the user does not need to quarantine for 14 days nor avoid contact with other people. This is important because not only it helps the person to understand his/her low risk of infection, but also reinforces how important it is to keep the protective measures against COVID-19; in (b), a yellow profile is shown representing a medium infection risk. Based on recent contact with suspected cases of COVID-19, the app tells the person that they might have been in contact with the virus, and therefore must practice social distancing for the next weeks, access essential services from home, and also inform close people about his/her risk; in (c), a red profile is shown representing a high infection risk. Based on recent contact with infected and suspected individuals, the app tells the user they must quarantine for the next 14 days and inform close people about his/her risk. This information is important to make sure that this individual does not put others at risk; in (d), a high risk of complication page is shown based on the previous conditions and comorbidities the user has. This kind of information helps the user to make better choices as regards his/her health, since they are more vulnerable and, therefore, more susceptible to become critically ill from COVID-19; in (e), a low risk of complication page is shown based on information provided by the user about his/her health. In this case, the user is not in one of the risk groups for COVID-19 and is less likely to present severe symptoms. Even though this might be the case for some users, the app still informs about the importance of following all the Health Ministry guidelines and keeping the protective measures for COVID-19. 
(a)

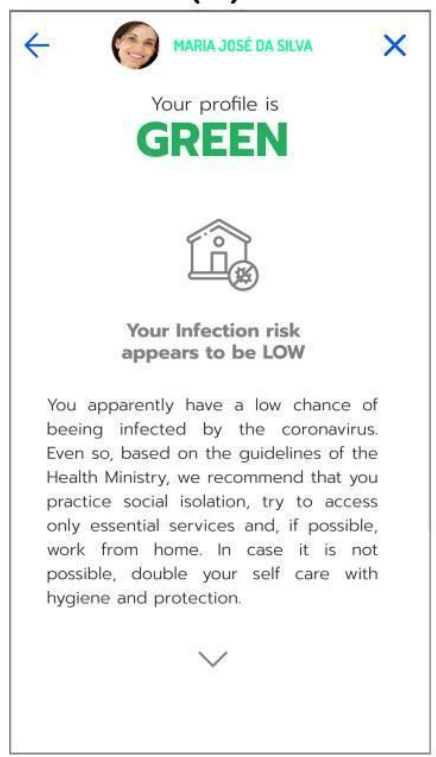

(b)

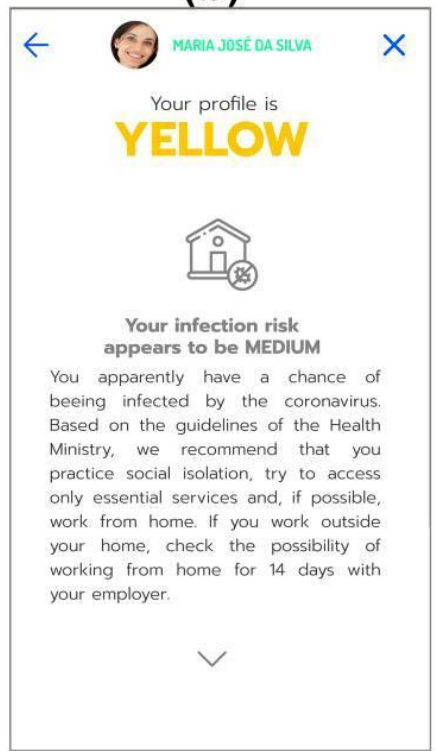

(c)

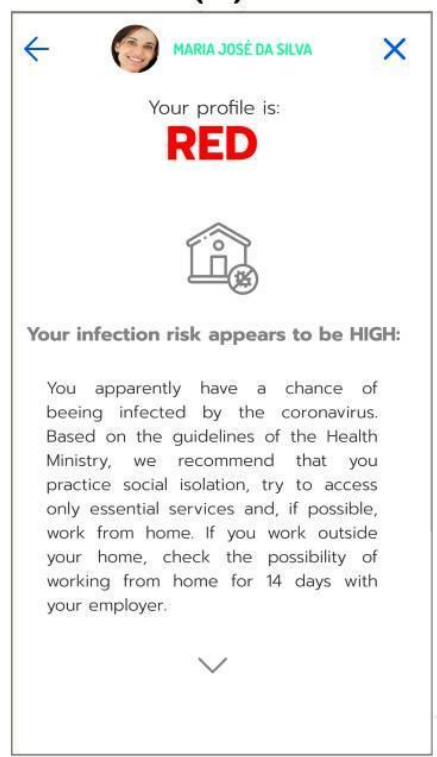

(e)

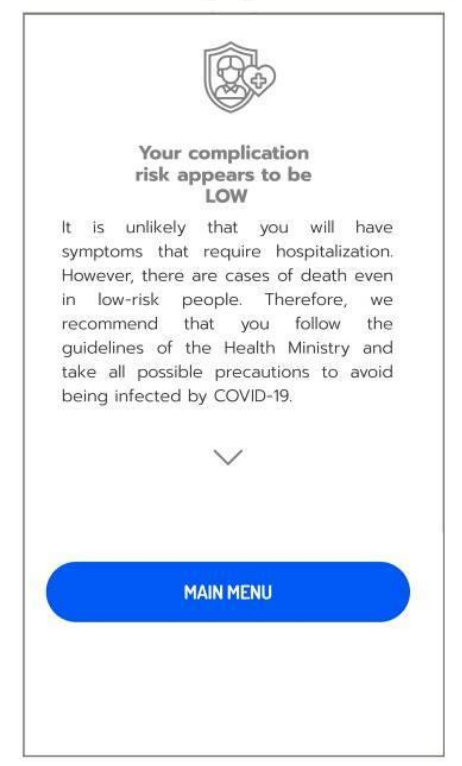

Figure 4 - Some of the information pages associated with Profile action. (a,b,c) low, medium and high infection risk; (d,e) low and high complication risk. 
"My Health" action on the main menu redirects to Figure 3(e), where the user can report a symptom and/or report a COVID-19 test result. This is important to estimate the risk of infection or to confirm it. While reporting a symptom, the user is requested to answer about its frequency (Figure 5a) and to report additional symptoms from a list (Figure 5b). This information is also important to calculate the risk of infection and differentiate mild cases from severe cases. If the user needs to report a COVID19 test result, he/she will be redirected to a page that asks about the result (Figure 5c) and when the test was taken (Figure 5d). The importance of knowing the result of the test and when it was taken is based on the possibility that the infected person might be able to spread the virus among others depending on how much time there is between the beginning of symptoms and the test result. 
(a)

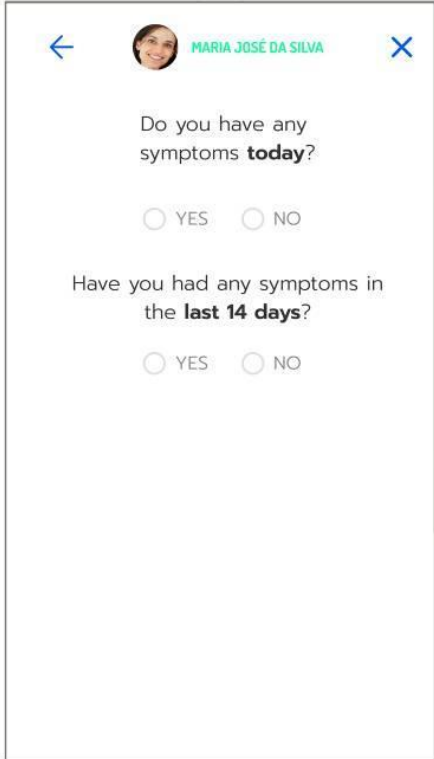

(c)

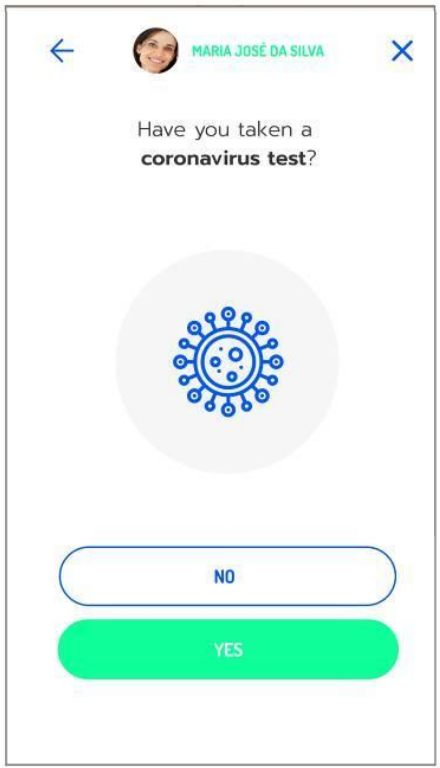

(b)

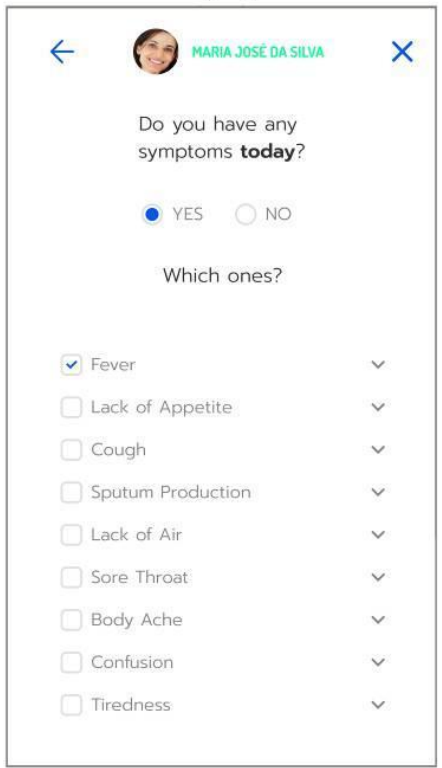

(d)

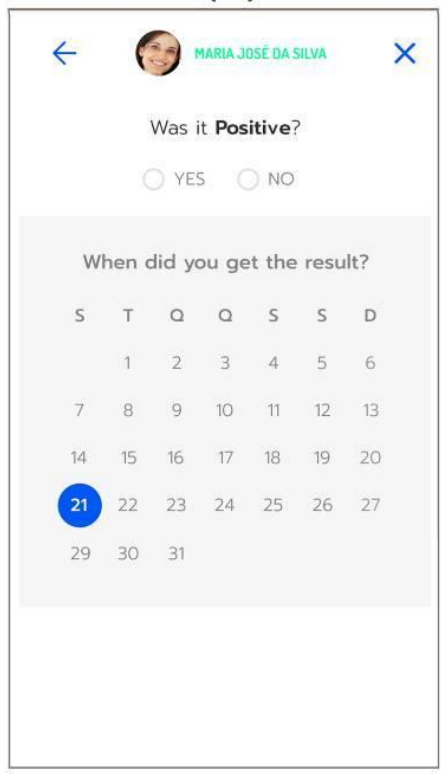

Figure 5 - My Health pages, where the user reports (a,b) symptoms and (c,d) test results. 
Health Agents: Type of Users

Three types of users are expected: Health Secretary's professionals, healthcare facility managers, and health professionals. The interface for each of these three users is described in the following subsections.

Health Secretary's User Navigation

Health Secretary's professionals will have access to the main page in Figure 6a, where they should register health establishments, see a heat map with the accurate density of infections (Figure 6b), see data from respective cities (Figure 6c), define criteria for tests and define quarantine criteria. Figure 6a shows a very simple and easy to understand main menu that can provide a fast experience for the Health Secretary's users to register health establishments and to access updated data about new cases in the city, which helps the public agents to understand the current epidemics' dynamic; in Figure 6b, it is possible to see a heat map for a region where the size of the circles is proportional to the number of infections in that area, also represented by the number in its middle. The colors help to identify the region's risk of infection, where orange represents medium risk and red, high risk; in Figure 6c, an example of municipality data is shown, which contains the mortality rate on the top left corner, followed by the immunization rate of the population, and the risk profile ratio. This ratio indicates the percentage of areas in the city that present a low, medium or high risk of infection. On the top center of the page, a demographic histogram is shown indicating the age ranges and their percentages in the population, which is important to calculate complication risks since the elderly are more vulnerable; then, a Reproduction Ratio graphic is shown on the bottom center of the page, a number that indicates if the pandemic is under control $\left(\mathrm{R}_{0}<1\right)$ or not $\left(\mathrm{R}_{0}>1\right)$. Finally, on the top right corner, a Daily New Cases graphic is shown so that professionals can visualize how new cases numbers vary in the time series, while on the bottom right corner a Total Cases graphic is shown, which calculates 
the accumulated number of cases in the city, which is important to estimate the population's immunization rate.
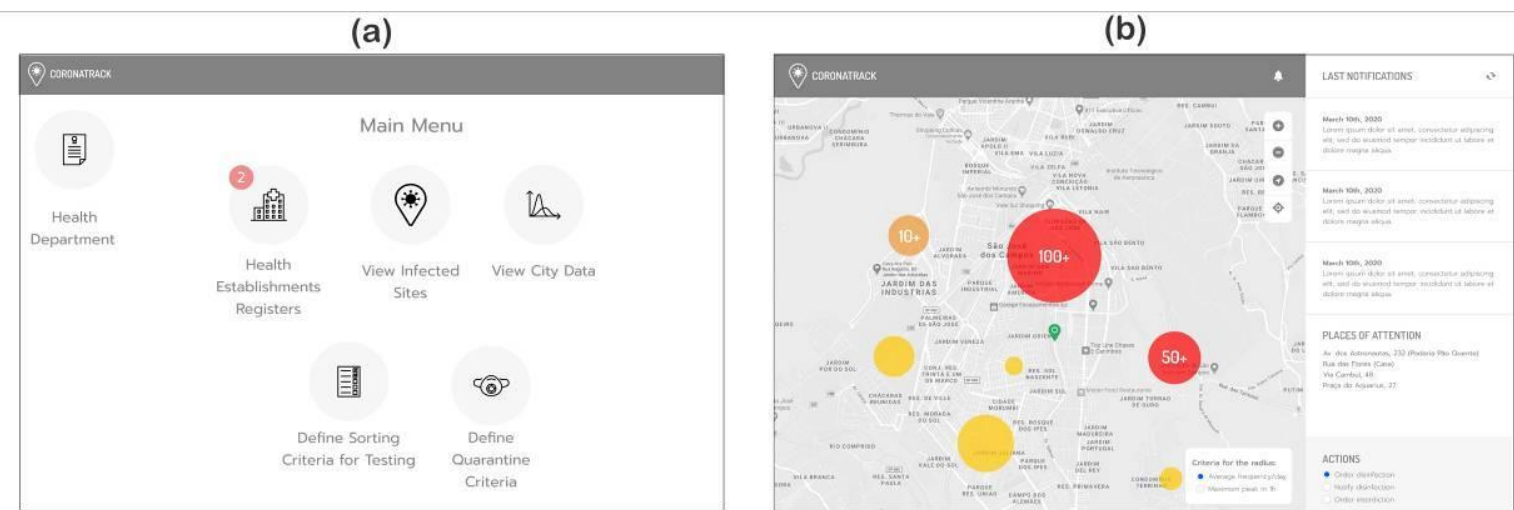

(c)

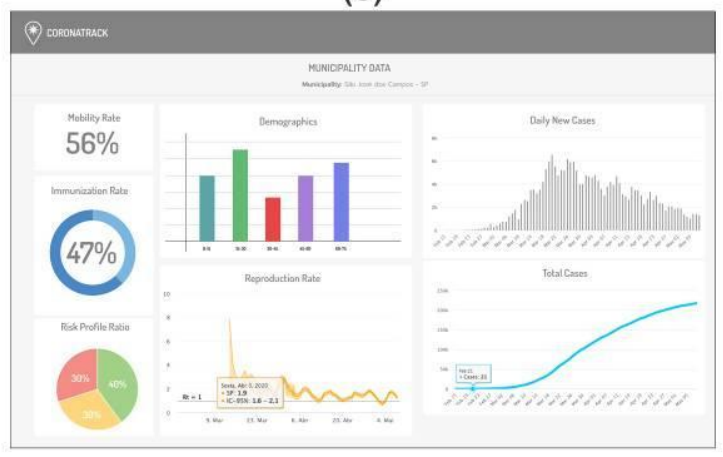

Figure 6 - (a) Health's Secretary agents' main page; (b) heat map; (c) municipality data page.

Healthcare Facility Manager Navigation

Healthcare facility managers, at their main page (Figure 7a), should register new Health professionals, select patients to take a COVID-19 test (Figure 7b), and see a heat map. Their main menu in Figure $7 \mathrm{a}$ is also very simple and easy to understand, making it faster for them to access needed information. In Figure $7 b$, it is shown how a user is selected to take a COVID-19 exam, where their names correspond with their comorbidities, infection risk, phone number, and IgM, IgG, and PCR exam results. All this information provides enough data to determine whether that user should take a COVID-19 exam. 
(a)

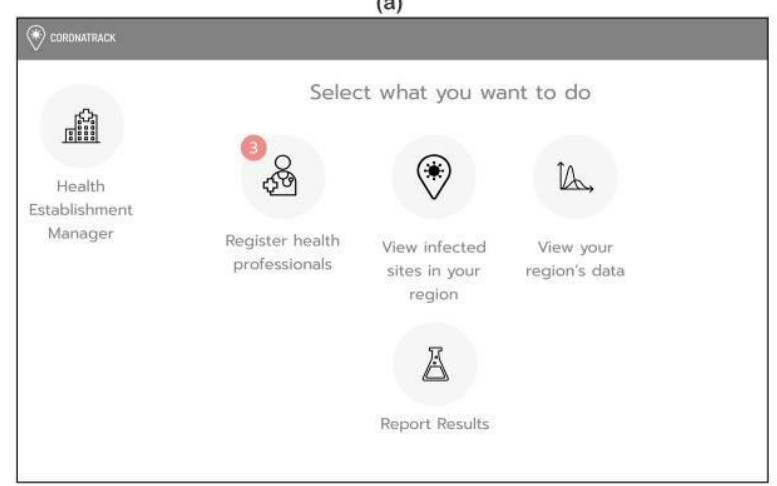

(b)

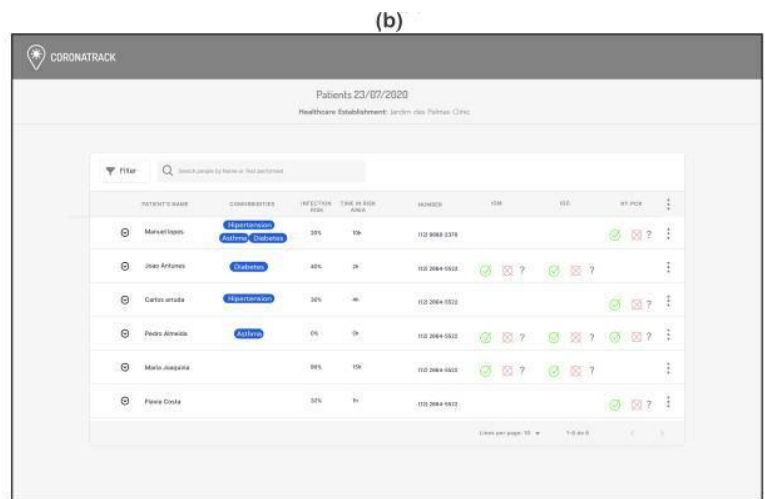

Figure 7 - (a) Healthcare facility manager's main page; (b) page with patients selected to take a COVID-19 test.

Health Professional Navigation

Health professionals access the application through the main page in Figure 8a, view scheduled tests accepted by their manager (Figure $8 \mathrm{~b}$ ), and report test results. Their main menu in Figure $8 \mathrm{a}$ is also very simple and easy to understand, making it faster for them to access needed information. In Figure $8 \mathrm{~b}$, their page is very similar to the page represented in Figure $7 \mathrm{~b}$ for Healthcare facility managers, but the difference is that they can only see which tests are scheduled to be performed and reported later.

(a)

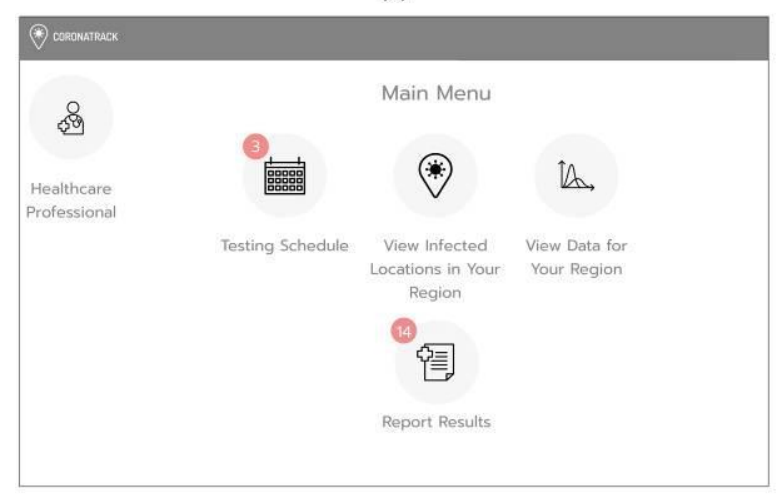

(b)

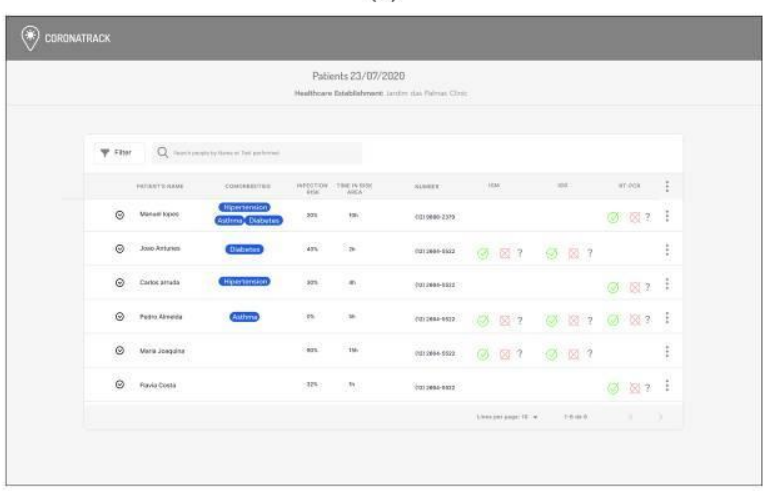

Figure 8 - (a) Health professionals' main page; (b) scheduled tests of COVID-19. 


\section{Discussion}

\section{Comparison with existing solutions}

This section compares CoronaTrack with other existing solutions, highlighting main limitations of these solutions, challenges imposed by the Brazilian reality (similar to the reality of several other countries around the world), and the set of solutions adopted by CoronaTrack to deal with these issues.

- Challenge \#1: Limited budget and logistical difficulties

Existing solutions are based on contact between people proven to be infected (through testing) and healthy people. CoronaTrack, on the other hand, identifies suspects based on a set of symptoms and changes the individual's risk profile based on that information, regardless of the availability of a test result. This is a very important contribution for countries like Brazil which have limited financial resources, but also for other countries which have faced difficulties in testing their population.

- Challenge \#2: Part of the population might not have access to a smartphone.

Existing contact-tracing solutions are based on the premise that there is the adherence of at least $30 \%$ of the population. Although this hypothesis holds true for developed countries, this is not a reality for developing countries. Nevertheless, even if there is no mass adherence of the population to the application, CoronaTack is able to use socio-demographic information to estimate the contact with infected people. This estimation is performed by using the individual's browsing history through densely populated spaces in places with high rates of infection. This is not the case for Brazil though, since official data shows that at least $79,1 \%$ of the population have access to the internet, and of those who had access to it, $99,2 \%$ declared using smartphones as the 
primary means of using the internet (IBGE, 2018). There are disparities among the 5 regions of the country, where the South, Southeast, and Midwest regions provide access to the internet to more than $80 \%$ of their population, whereas only $72,1 \%$ of the population from the North region and $69,1 \%$ from the Northeast region can access it (IBGE, 2018). These disparities are even greater when comparing the urban and rural populations, with the rural communities generally scoring 30 percentage points less than the urban ones (IBGE, 2018). Even though this is true for all the Brazilian territory, it is known that COVID-19 impacted more the urban zones in the country, where the pandemic imposed a great loss of lives and economic restrictions, so it is possible to say that the CoronaTrack solution proposed here still represents a reasonable approach to fight the pandemic specifically in urban areas where there is wide use of smartphones by the population.

- Challenge \#3: Size of the population and difficulty to disseminate the solution Available Heat-Map-based solutions use only information from users of the application and are based on the premise that there will be a bulk adhesion to the application. However, countries with larger populations and greater territories may face difficulties in disseminating solutions of this nature and encouraging individuals to install an application. To solve this issue, CoronaTrack displays a Heat Map based on both census georeferenced information and application user data, allowing individuals who need to move around the municipality to avoid zones with a high density of suspects and infected people (hot zones).

- Challenge \#4: Logistical difficulties in providing care, as well as applying tests and vaccines to the population

The available solutions do not help the health agent to carry out the prioritization work. CoronaTrack, on the other hand, offers an interface that allows the health worker to view the 
epidemiological profile of the municipality; summon patients to perform tests, get a vaccine or receive teleorientation; and capture records of external campaigns of health agents.

\section{High quality of health information communication}

Almost $80 \%$ of the population in Brazil has access to the internet from smartphones, showing that a major part of the population can have access to online health information, which is not always safe nor accurate (IBGE, 2018). Studies showed that trust is an important factor in health knowledge adoption by the population during high health threats (Huo et al., 2017), and that in countries with lower socioeconomic indicators and greater social disparities, such as Brazil, the population searches for information on less accurate platforms and takes longer to find relevant and helpful health knowledge on them (Chen et al., 2020; Rantala et al., 2019). Considering the recent conflicts between the federal government and state governors in respect to COVID-19 safety measures and the denial of the virus' threat by the federal authorities (Aquino et al., 2020; Stotopoli et al., 2020), it is possible to say that health information about the pandemic in Brazil has been misrepresented, leading part of the population to discredit safety measures, the quality of possible vaccines and the global recommendations to stop COVID-19 from spreading. The results presented in this work show that the CoronaTrack is an application that can collect updated data on testing, new cases and deaths from COVID-19 very easily and fast, calculate infection risk, generate heat maps and provide individualized information about the user's risk of infection and complication, orientating about selfcare actions. These features are in accordance with studies that show how smartphone applications can be a helpful tool to inform consumers about their health risks and to give orientation on self-care behaviors (Rathbone et al., 2017). Therefore, the CoronaTrack is a possible solution to make the communication of health information more accurate, verified, and safe for the population, helping to combat false information that presents a risk to collective health during a pandemic. 


\section{Conclusion}

This paper presented the specification and implementation of an Information System for Epidemic Control developed to combat the COVID-19 pandemic outbreak. The solution was inspired by worldwide successful experiences in the usage of technology to minimize the negative consequences of such pandemic, as well as on lessons learned from non-effective solutions, addressing the main challenges in this domain. The solution was validated by a comprehensive and representative multidisciplinary team.

Four original features were implemented to fill the gaps between existing automatic Contact Tracing solutions and the Brazilian reality challenges, as follows: (i) identification of infection suspects and revision of user infection risk based on recent symptoms records; (ii) inference of infection risk based on the user navigation history; (iii) Heat Map construction based on the combination of permanent residence census information and user georeferenced location; (iv) healthcare agent interface that allows epidemic dashboards visualization, patient call to perform a test, receive vaccine or teleorientation, as well as healthcare agents campaign actions records.

As other countries and scenarios of fighting against a pandemic have very similar challenges, these original features would definitively increase the probability of success of such a solution in the epidemic control process. The CoronaTrack not only receives updated data on new cases, new deaths, and testing results to generate heat maps and calculate the risk of infection in specific regions, serving as a guide for public agents to orientate public policies to fight against a pandemic, but it also works as a safe and easy-to-understand platform that provides accurate health information to the population, helping to build trust in the governmental policies to manage the sanitary crisis, which represents a high-level threat to people's lives. Knowing that the users' search for health information on the internet works as a learning process about their risks and individual responsibilities in containing the virus, the CoronaTrack represents a potential solution to combat false information about the pandemic, since it provides safe, accurate, and verified health information about COVID19. In fact, and most importantly, the complete solution can be easily adapted to become a generic 
Information System for Epidemic Control that can control other epidemic diseases, like Zika Virus, measles, H1N1, and many others. This potential applicability of the app for other contagious diseases can have a great positive impact in the Brazilian sanitary scenario, which faces challenges to contain epidemics other than COVID-19 on a yearly basis.

The initiative has the potential to become a reference in Brazil on how to coordinate the efforts of health authorities and the population on any epidemic control. On the other hand, some issues still need to be investigated. It should be studied if a more comprehensive and invasive solution would be well accepted by the general population, even knowing that all sensitive user data are protected; the effectiveness of the solution in terms of changing the general population mobility habits should be analyzed; and the impact of each solution feature on the population behavior should be quantified. More research needs to be performed to investigate these open questions.

\section{Acknowledgments}

H. M. Paiva, L. F. A. Silva and D. G. Sanches acknowledge the support of São Paulo Research Foundation FAPESP (grants \#2019/18294-7 and \#2020/08770-3).

The other authors received no specific funding for this work.

The funders had no role in study design, data collection and analysis, decision to publish, or preparation of the manuscript.

\section{References}

Abebe, E. C., Dejenie, T. A., Shiferaw, M. Y., and Malik, T. (2020). "The newly emerged COVID19 disease: a systemic review". Virology journal, Vol. 17, No. 1, pp. 1-8. doi:10.1186/s12985-02001363-5. 
Aquino, E. M. L., Silveira I. H., Pescarini J. M., Aquino, R. et al. (2020). "Social distancing measures to control the COVID-19 pandemic: potential impacts and challenges in Brazil". Ciência \& Saúde Coletiva, Vol. 25, p. 2423-2446, doi: 10.1590/1413-81232020256.1.10502020.

Bradford, L. R., Aboy, M., and Liddell, K. (2020). "COVID-19 Contact Tracing Apps: A Stress Test for Privacy, the GDPR and Data Protection Regimes". Journal of Law and the Biosciences.

Brazilian Ministry of Health (2020), "Guidelines for the diagnosis and treatment of COVID-19" (in Portuguese), available at: https://coronavirus.saude.gov.br/manejo-clinico-e-tratamento (accessed 25 October 2020).

Camc1, B., Ersoy, C., and Kaynak, H. (2019). "Abnormal respiratory event detection in sleep: a prescreening system with smart wearables”. Journal of Biomedical Informatics, Vol. 95, p. 103218. doi: 10.1016/j.jbi.2019.103218.

Chen, Y., Zhao, Y., and Wang, Z. (2020), "Understanding online health information consumers' search as a learning process: Search platform sequences, query reformulation and conceptual changes", Library Hi Tech, Vol. 38 No. 4, pp. 859-881. doi: 10.1108/LHT-08-2019-0174.

Chinese Government (2020). "China colored health code", available at: http://newyork:chineseconsulate:org/chn/fwzc/zxtz/t1769212:htm (accessed 05 October 2020).

D’Amico, F., Baumgart, D. C., Danese, S., and Peyrin-Biroulet, L. (2020). “Diarrhea during COVID19 infection: pathogenesis, epidemiology, prevention and management". Clinical Gastroenterology and Hepatology, Vo. 18, No. 8, pp. 1663-1672. doi: 10.1016/j.cgh.2020.04.001.

Dudden A, and Marks A. (2020) "South Korea took rapid, intrusive measures against COVID-19 and they worked". The Guardian, available at: https://www.theguardian.com/commentisfree/2020/mar/20/south-korea-rapid-intrusive-measurescovid-19 (accessed 05 October 2020). 
Ferretti, L., Wymant, C., Kendall, M., Zhao, L., Nurtay, A., Abeler-Dörner, L., Parker M,, Bonsall D,, and Fraser C. (2020). "Quantifying SARS-CoV-2 transmission suggests epidemic control with digital contact tracing”. Science, Vol. 368, No. 6491. doi: 10.1126/science.abb6936.

Figma: the collaborative interface design tool. www.figma.com. Last access on Oct 5th, 2020.

Hong Kong Government (2020). "StayHomeSafe Mobile App User Guide", available at: https://www.coronavirus.gov.hk/eng/stay-home-safe.html (accessed 05 October 2020).

Hu, Y., Sun, J., Dai, Z., Deng, H, Li, X., Huang, Q., Wu, Y., Sun, L., and Xu, Y. "Prevalence and severity of coronavirus disease 2019 (COVID-19): A systematic review and meta-analysis". Journal of Clinical Virology, p. 104371. doi: 10.1016/j.jcv.2020.104371.

Huo, C., Zhang, M., and Ma, F. (2018), "Factors influencing people's health knowledge adoption in social media: The mediating effect of trust and the moderating effect of health threat", Library Hi Tech, Vol. 36, No. 1, pp. 129-151.

IBGE - Brazilian Institute of Geography and Statistics (2018), "Access to internet, television and mobile telephony". In: National Household Sample Survey (in Portuguese). Available at: https://ftp.ibge.gov.br/Trabalho_e_Rendimento/Pesquisa_Nacional_por_Amostra_de_Domicilios_co ntinua/Anual/Acesso_Internet_Televisao_e_Posse_Telefone_Movel_2018/Analise_dos_resultados_T IC_2018.pdf (accesses 24 Dec 2020).

Iyengar, K., Bahl, S., Vaishya, R., and Vaish, A. (2020). "Challenges and solutions in meeting up the urgent requirement of ventilators for COVID-19 patients". Diabetes \& Metabolic Syndrome: Clinical Research \& Reviews. Vol. 14, No. 4, pp. 499-501. doi: 10.1016/j.dsx.2020.04.048.

Jiang, P., Fu, X., Van Fan, Y., Klemeš, J. J., Chen, P., Ma, S., and Zhang, W. (2020). "Spatialtemporal potential exposure risk analytics and urban sustainability impacts related to COVID-19 
mitigation: A perspective from car mobility behavior". Journal of Cleaner Production, Vol. 279, p. 123673. doi: 10.1016/j.jclepro.2020.123673.

Johnson, C. M., McIlwain, S., Gray, O., Willson, B., and Vorderstrasse, A. (2017). "Creating a sustainable collaborative consumer health application for chronic disease self-management". Journal of Biomedical Informatics, Vol. 71, pp. 198-206. doi: 10.1016/j.jbi.2017.06.004.

Khai, H., and Kai M. (2020). "Interactions of coronaviruses with ACE2, angiotensin II, and RAS inhibitors-lessons from available evidence and insights into COVID-19". Hypertension Research, Vol. 43, No. 7, pp. 648-654. doi: 10.1038/s41440-020-0455-8.

Kucharski AJ, Russell TW, Diamond C, Liu Y, Edmunds J, Funk S, Eggo RM; Centre for Mathematical Modelling of Infectious Diseases COVID-19 working group. Early dynamics of transmission and control of COVID-19: a mathematical modelling study. Lancet Infect Dis. 2020 May;20(5):553-558. doi: 10.1016/S1473-3099(20)30144-4. Epub 2020 Mar 11. Erratum in: Lancet Infect Dis. 2020 Mar 25;: PMID: 32171059; PMCID: PMC7158569.

Kumar, M., and Mostafa, J. (2020), "Electronic health records for better health in the lower- and middle-income countries: A landscape study", Library Hi Tech, Vol. 38 No. 4, pp. 751-767. doi: 10.1108/LHT-09-2019-0179

Levy, I. (2020). "High level privacy and security design for NHS COVID-19 Contact Tracing App". National Cyber Security Centre, UK, available at: https://www.ncsc.gov.uk/files/NHS-app-securitypaper\%20V0.1.pdf (accessed 05 October 2020).

Mao, Y., Jiang, S., and Nametz, D. "Data-driven Analytical Models of COVID-2019 for Epidemic Prediction, Clinical Diagnosis, Policy Effectiveness and Contact Tracing: A Survey”. Preprints 2020, 2020070124 (doi: 10.20944/preprints202007.0124.v1). 
Masood, A., Sheng, B., Li, P., Hou, X., Wei, X., Qin, J., and Feng, D. (2018). "Computer-Assisted Decision Support System in Pulmonary Cancer detection and stage classification on CT images". Journal of biomedical informatics, Vol. 79, pp. 117-128. doi: 10.1016/j.jbi.2018.01.005.

Navarro-Alamán, J., Lacuesta, R., Garcia-Magariño, I., and Gallardo, J. (2020). “A methodology for the design and development of gamified mobile apps for monitoring cancer survivors". J Journal of Biomedical Informatics, Vol. 106, p. 103439. doi: 10.1016/j.jbi.2020.103439.

Newman, N., Gilman, S., Burdumy, M., Yimen, M., and Lattouf, O. (2020). "A Novel Tool for Patient Data Management in the ICU—Ensuring Timely and Accurate Vital Data Exchange Among ICU Team Members". International Journal of Medical Informatics, Vol. 144, p. 104291. doi: 10.1016/j.ijmedinf.2020.104291

Nicola M, Alsafi Z, Sohrabi C, et al. The socio-economic implications of the coronavirus pandemic (COVID-19): A review. Int J Surg. 2020;78:185-193. doi:10.1016/j.ijsu.2020.04.018

Nikel, D. (2020) “Smittestopp: How Norway’s New Coronavirus App Works. Life in Norway", available at: https://www:lifeinnorway:net/smittestopp-coronavirusapp/ (accessed 05 October 2020).

Parasa, S., Desai, M., Chandrasekar, V. T., Patel, H. K., Kennedy, K. F., Roesch, T.,Spadaccini, M., Colombo, M., Gabbiadini, R., Artifon, E. L. A., Repici, A., Sharma, P. "Prevalence of Gastrointestinal Symptoms and Fecal Viral Shedding in Patients With Coronavirus Disease 2019: A Systematic Review and Meta-analysis". JAMA Network Open, Vol. 3, No. 6, pp. e2011335e2011335. doi: 10.1001/jamanetworkopen.2020.11335.

Pashazadeh, A., and Navimipour, N. J. (2018). "Big data handling mechanisms in the healthcare applications: A comprehensive and systematic literature review". Journal of Biomedical Informatics, Vol. 82, pp. 47-62. doi: 10.1016/j.jbi.2018.03.014. 
Rantala, A., Enwald, H. and Zinn, S. (2019), "Web-based health information seeking: A small-scale comparative study between Finnish and South African university students", Library Hi Tech, Vol. 37 No. 4, pp. 933-944. doi: 10.1108/LHT-08-2018-0109

Rathbone AL, Prescott J. (2017) "The Use of Mobile Apps and SMS Messaging as Physical and Mental Health Interventions: Systematic Review". Journal of medical Internet research, v. 19, n. 8. doi: 10.2196/jmir.7740.

Singapore Ministry of Health (2020). "How TraceTogether works", available at: https://www.tracetogether.gov.sg/ (accessed 05 October 2020).

Sligo J., Gauld R., Roberts V., and Villa, L. (2017). "A literature review for large-scale health information system project planning, implementation and evaluation". International Journal of Medical Informatics, Vol. 97, pp. 86-97.

Storopoli J, Braga da Silva Neto WL, Mesch GS. (2020) "Confidence in social institutions, perceived vulnerability and the adoption of recommended protective behaviors in Brazil during the COVID-19 pandemic". Social Science \& Medicine, Vol. 265, paper 113477. doi: 10.1016/j.socscimed.2020.113477.

Williams L., and Cockburn A. (2003). “Agile software development: it's about feedback and change”. IEEE Computer, Vol. 36, No. 6, pp. 39-43.

Zastrow M. (2020). "Coronavirus contact-tracing apps: can they slow the spread of COVID-19", Nature Technology Features. doi: 10.1038/d41586-020-01514-2 